\title{
Exciton Delocalization in the B850 Light-Harvesting Complex: Comparison of Different Measures
}

\author{
M. Dahlbom, ${ }^{\dagger}$ T. Pullerits, ${ }^{\dagger}$ S. Mukamel, ${ }^{*, *}$ and V. Sundström $*, \dagger$ \\ Department of Chemical Physics, Lund University, Box 124, 21100 Lund, Sweden, and \\ Department of Chemistry, University of Rochester, Rochester, New York 14627
}

Received: December 14, 2000; In Final Form: March 19, 2001

\begin{abstract}
A much debated topic in recent studies of ultrafast exciton dynamics in molecular aggregates has been the precise definition of the exciton coherence domain in the B850 aggregate of photosynthetic purple bacteria. In this work we compare several measures of exciton delocalization and their variation with temperature, inhomogeneous broadening, and time. We find that the exciton wave packet prepared by a $40 \mathrm{fs}$ pulse is initially delocalized over a substantial portion of the ring and contracts to a significantly smaller size on the time scale of a few hundreds of femtoseconds. Steady-state absorption and time-dependent pump-probe spectra of the B850 aggregate are simulated and compare well with previously reported experimental data. The calculated thermalized exciton wave packet size is in good agreement with previous experimental and theoretical estimates.
\end{abstract}

\section{Introduction}

Molecular aggregates belong to a family of molecular nanostructures that lies between crystals and isolated molecules. J-aggregates, ${ }^{1}$ polymers, ${ }^{2}$ photosynthetic antenna complexes, ${ }^{3}$ and dendrimers ${ }^{4,5}$ are a few examples of such systems. As the number of chromophores in the aggregate $(N)$ is increased, the system properties change from molecular to bulk type, and sizedependent effects must be considered in aggregates, including energy transfer, resonance interaction, exchange narrowing, and collective spontaneous emission (superradiance). Collective excited states, excitons, are formed in molecular crystals and aggregates due to strong coupling. ${ }^{6,7}$ In an ideal system, without exciton-phonon interactions and static disorder, the molecular exciton states would be perfectly delocalized over the entire aggregate. However, various perturbations may localize these states to a part of the aggregate. There are a number of localization mechanisms to consider: static (on-site energetic and orientation) disorder, exciton-phonon coupling (dynamic disorder and polaron formation) and impurities. In onedimensional molecular aggregates the delocalization of the exciton can be well described by a length scale, $L_{\mathrm{d}}$, i.e., the number of molecules that share the excitation. In general, however, higher dimensionalities or fractal structures necessitate a broader definition of an exciton delocalization domain (ECD). ${ }^{8,9}$

The ECD of Frenkel excitons lends itself to a simple real space visualization, enabling intuitive interpretation of excitation dynamics and a deeper understanding of how interaction strength and homogeneous and inhomogeneous broadening contribute to the spectroscopic properties of the molecular aggregate.

Photosynthetic antenna systems have been used as prototypes for studies of exciton effects. ${ }^{10}$ Recently, the structure of the peripheral light-harvesting complex (LH2) of photosynthetic purple bacteria was determined to atomic resolution for

\footnotetext{
$\dagger$ Lund University.

$\div$ University of Rochester.
}

Rhodopseudomonas (Rps.) acidophila ${ }^{11}$ and Rhodospirillium molischianum. ${ }^{12}$ LH2 contains two concentric rings of bacteriochlorophyll (BChl) molecules named B800 and B850 after their characteristic $\mathrm{Q}_{y}$ absorption maxima at 800 and $850 \mathrm{~nm}$ (see Figure 1). Surrounding the BChl pigments is a cylindrical "cage" formed by $\alpha$ and $\beta$ protein helicies. In Rps. acidophila, the B800 ring consists of 9 well-separated BChl molecules, roughly 18 $\AA$ apart, with excited states having a localized character. ${ }^{13}$ Contrary to that, the $18 \mathrm{BChl}$ molecules of the $\mathrm{B} 850$ ring are densely packed with center-to-center distances of about $9 \AA$, and strong intermolecular interaction leads to a considerable delocalization of the excitations. Various experimental and theoretical techniques have been employed to characterize the exciton, ${ }^{14-33}$ and the resulting values of $L_{\mathrm{d}}$ range from a few pigment molecules ${ }^{15-19}$ to the entire ring. ${ }^{31,32}$ In this paper we will show that the large variation in $L_{\mathrm{d}}$ obtained by different authors is the result of different definitions of the exciton delocalization, as well as different experimental probes for exciton coherence.

Localization properties of individual exciton eigenstates can be defined and analyzed. However, in most experiments the signals depend on superpositions of many such eigenstates, making it impossible to connect the individual eigenstates directly with observables. Mukamel and co-workers have developed descriptions of optical signals of chromophore aggregates based on the excitonic density matrix $N_{m n}$ (eq 2.6). ${ }^{34-36}$ These include nonlinear susceptibilities (frequency domain) $)^{34,35}$ as well as pump-probe, fluorescence, and cooperative spontaneous emission (superradiance). ${ }^{14,15,21,36-39}$ An electron-hole density matrix has been also used to measure the coherence size in conjugated polymers. ${ }^{2,4,40}$ The diagonal elements of the density matrix $N_{n n}$ (in the real space, chromophore, representation) give the populations of the various chromophores. However, more interesting is to look at the antidiagonal directions, i.e., the variation of $N_{m n}$ with $n-m \cdot{ }^{35,36}$ The density matrix is the most natural way of characterizing the coherence size, and the exciton density matrix provides a 


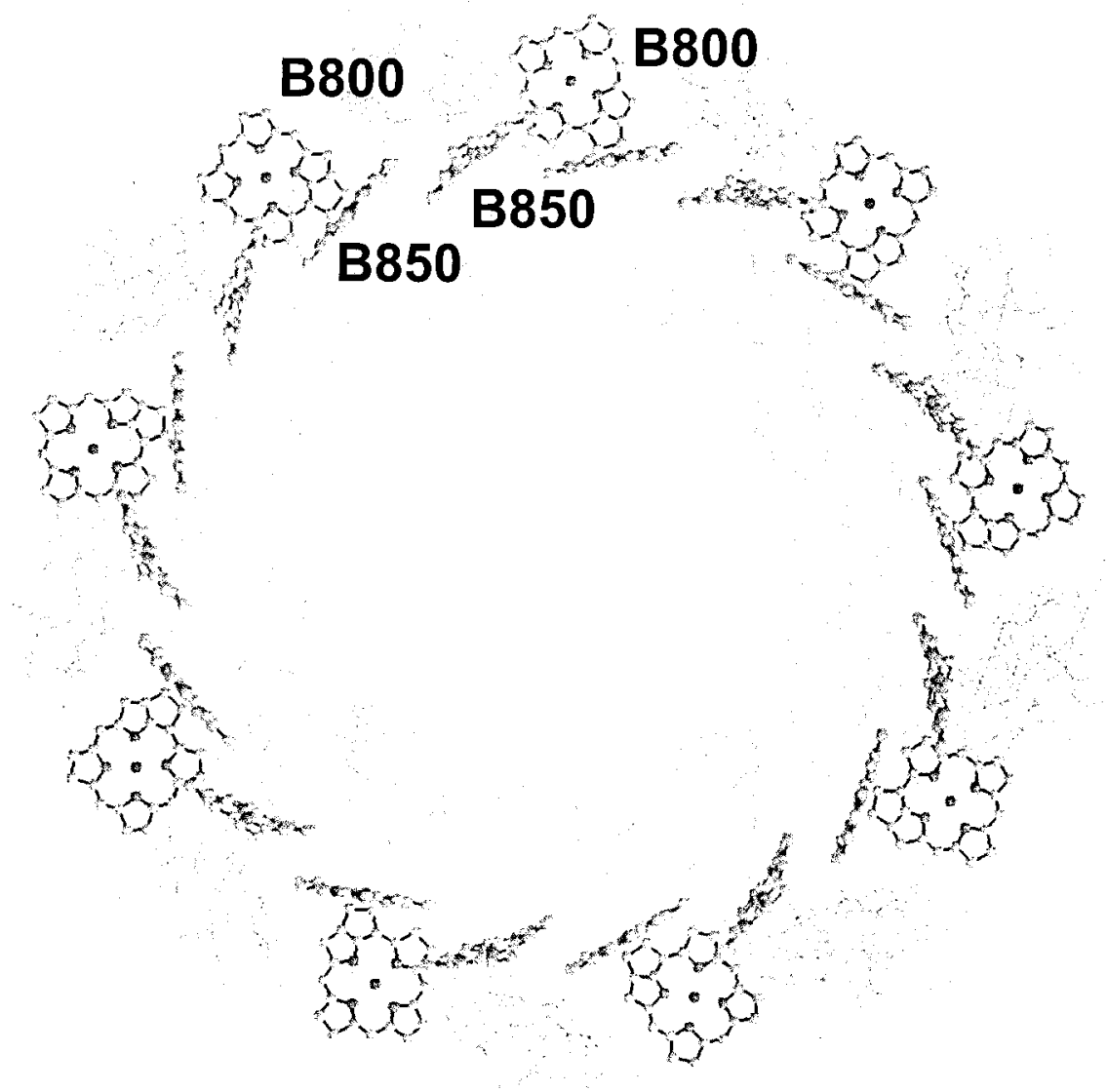

Figure 1. Two concentric BChl rings in the LH2 complex of Rps. acidophila seen in their protein environment. The pigments parallel to the surface belong to B800 and the perpendicular to B850.11

direct measure of exciton coherence that is closely connected to experimental signals such as pump- probe ${ }^{15,21}$ and cooperative spontaneous emission superradiance. ${ }^{41}$ It is reasonable to assume that the initial exciton wave packet created by a femtosecond pulse is delocalized over a substantial part of the ring and through the mechanisms described earlier it rapidly becomes localized on a smaller set of chromophores. ${ }^{15,20,42}$

In this paper we simulate the time dependent localization of the B850 exciton as reflected in experimental transient absorption and superradiance measurements. We further compare several definitions of $L_{\mathrm{d}}$ and examine their differences. The article is organized as follows: In section II, we present the Frenkel exciton model for the aggregate and express the pumpprobe spectroscopy and superradiance in terms of the exciton density matrix. The various definitions of $L_{\mathrm{d}}$ are given and discussed in section III. Numerical calculations are presented in section IV, and the conclusions are drawn in section V.

\section{The Exciton Density Matrix in Pump-Probe Spectroscopy and Superradiance Experiments}

In this section we briefly review the model that describes the B850 aggregate as a set of coupled three-level systems. We discuss the real space doorway-window formulation of ultrafast pump-probe spectroscopy and superradiance. The time-dependent exciton density matrix will be used to calculate the different exciton delocalization length estimates defined in the following section. ${ }^{14,43}$

The B850 aggregate has a 9-fold ring symmetry consisting of 18 weakly dimerized BChl(a) molecules (Figure 1). The model chromophores have a ground state $\left(\mathrm{S}_{0}\right)$, a first excited state $\left(S_{1}\right)$, and a second excited state $\left(S_{2}\right)$. The ground-state absorption spectrum corresponds to the $S_{0} \rightarrow S_{1}$ transition, the so-called $\mathrm{Q}_{y}$ transition. In our calculations we use a Green function formulation of the optical response functions in the real-space doorway-window representation. ${ }^{14,44}$ The calculation can be divided into three steps: First, (i) the pump pulse excites an exciton wave packet, the doorway, in the system. During the pulse delay this initial wave packet then interacts with the surrounding environment (step ii). Finally, (step iii) the probe pulse creates a second wave packet, the window, and the Liouville space overlap with the propagated doorway wave packet results in the pump-probe signal. The doorway-window expressions use the Wigner spectrogram representation of the fields. ${ }^{44,50,51}$ This mixed time and frequency representation is well suited for the spectroscopic simulations performed in this paper since pulses of arbitrary shapes and durations can be used.

The electronic motions are described by the Frenkel Hamiltonian, which in the Heitler-London approximation reads, ${ }^{45}$

$$
H=\sum_{m n} h_{m n} \hat{B}_{m}^{\dagger} \hat{B}_{n}+\sum_{n} \frac{g_{n}}{2}\left(\hat{B}_{n}^{\dagger}\right)^{2}\left(\hat{B}_{n}\right)^{2}-E(t) \cdot P
$$

$\hat{B}_{m}^{\dagger}\left(\hat{B}_{m}\right)$ are the exciton creation (annihilation) operators that create (remove) an excitation on the $m$ th chromophore, satisfying the commutator relations

$$
\left[\hat{B}_{n}, \hat{B}_{m}^{\dagger}\right]=\delta_{m n}\left(1-\left(2-\kappa_{n}^{2}\right) \hat{B}_{n}^{\dagger} \hat{B}_{n}\right)
$$


The Hamiltonian matrix elements are $h_{m n}=\left(E_{n} \delta_{\mathrm{mn}}+J_{\mathrm{mn}}\right)$ and $g_{n} \equiv 2 \hbar\left(\left(2-\kappa_{n}{ }^{2}\right) \Omega_{n}+\Delta_{n}\right) / \kappa_{n}^{2}$ is the nonlinearity parameter. The energetic offset of the chromophores from a harmonic threelevel structure is measured by $\Delta_{n}$ so that the energy gap between the $\mathrm{S}_{2}$ and $\mathrm{S}_{1}$ levels is $\left(\Omega_{n}^{(2)}-\Omega_{n}^{(1)}=\left(\Omega_{n}+\Delta_{n}\right) \cdot g_{n}\right.$ contain all the information about the two-exciton state manifold and thus we can avoid explicit calculation of this manifold. $\kappa_{n}$ is the ratio of the $S_{1} \rightarrow S_{2}$ and $S_{0} \rightarrow S_{1}$ transition dipoles of the individual chromophores. ${ }^{45}$ The polarization operator, which controls the interaction term between the system and the external field, $E(t)$ reads

$$
P=\sum_{n} \mu_{n}\left(\hat{B}_{n}^{\dagger}+\hat{B}_{n}\right)
$$

We further assume that the electronic system is linearly coupled to a bath of harmonic oscillators representing the surrounding environment. The collective coordinates of the nuclear oscillators are eliminated using projection operator techniques, ${ }^{46}$ and the bath influence on the electronic system is completely contained in the Redfield relaxation superoperator. ${ }^{46-49}$

The one-exciton energies and wavevectors can be obtained from the eigenrelation $\sum_{n} h_{m n} \varphi_{\alpha}(n)=\epsilon_{\alpha} \varphi_{\alpha}(m)$ and the transition dipole in the exciton representation is then $\mu_{\alpha}=\sum_{m} \varphi_{\alpha}(m) \mu_{m}$. Using the exciton basis, the linear absorption spectrum is given by ${ }^{14}$

$$
S_{\mathrm{abs}}(\omega)=\sum_{\alpha} \frac{2\left|\mu_{\alpha}\right|^{2} \epsilon_{\alpha}}{\left(\omega-\epsilon_{\alpha}\right)^{2}+\Gamma^{2}}
$$

where $\Gamma$ is a phenomenological electronic dephasing rate, corresponding to the half-width at half-maxima of the Lorentzian line-shape function.

The pump-probe signal in the doorway-window representation was calculated by solving the nonlinear exciton equations $(\mathrm{NEE}),{ }^{45}$

$$
\begin{aligned}
& S_{p p}\left(\tau, \bar{\omega}_{2}, \bar{\omega}_{1}\right)= \\
& \quad \sum_{m n} \int_{0}^{\infty} \mathrm{d} \tau^{\prime} \int_{0}^{\infty} \mathrm{d} t_{2} \mathrm{~W}_{m n}\left(\tau-\tau^{\prime}, \bar{\omega}_{2}\right) N_{m n}\left(\tau^{\prime}-t_{2}, \bar{\omega}_{1}\right)
\end{aligned}
$$

The exciton density matrix (EDM), is defined as

$$
N_{m n}(t)=\left\langle\hat{B}_{m}^{\dagger}(t) \hat{B}_{n}(t)\right\rangle
$$

Following the interaction with the pump, the time-dependent exciton density matrix can be represented by the propagated doorway function defined as

$$
N_{m n}\left(\tau^{\prime}-t_{2}, \bar{\omega}_{1}\right) \equiv \sum_{k l} \bar{G}_{\mathrm{mn}, k l}\left(t_{2}\right) \mathrm{D}_{k l}\left(\tau^{\prime}-t_{2}, \bar{\omega}_{1}\right)
$$

where the initial exciton density matrix is given by the doorway function. Expressions for the doorway (D ) and window functions (W), dressed with the Wigner spectrograms of the pulses, ${ }^{50,51}$ are given in ref 44 . The time arguments $\tau, \tau^{\prime}$, and $t_{2}$ are related to the time delay between the pump and the probe pulses, and the overall pulse characteristics, whereas $\bar{\omega}_{1}$ and $\bar{\omega}_{2}$ are the central frequencies of the pump and probe pulses. The superoperator $\bar{G}(t)$ represents the evolution of the exciton density matrix. It incorporates the phonon bath influence on the system using the Redfield relaxation superoperator. ${ }^{48,49,52}$ Each molecule is coupled to an uncorrelated-oscillator bath, which is modeled using the spectral density of the overdamped Brownian oscillator, ${ }^{14,46}$ see ref 44 for details.
Another observable, the superradiance enhancement factor, which represents the radiative decay rate of the aggregate relative to that of the monomer, is given by ${ }^{15}$

$$
L_{s}(\tau)=\sum_{m n} M_{m n} N_{m n}(\tau)
$$

The window function is given by $M_{m n}=\mathbf{d}_{m} \cdot \mathbf{d}_{n}$ and contains all the relevant geometric information about the system. Here $\mathbf{d}_{n}$ is the unit dipole vector of the $n$th chromophore. Superradiance offers perhaps the most direct connection between an experimental observable and the EDM. When all dipoles are parallel (a linear aggregate), $M_{m n}=1$, and $L_{\mathrm{s}}$ provides a direct estimate of the exciton delocalization length. Otherwise $L_{\mathrm{S}}$ shows an interplay of geometry and exciton dynamics. Thus, the superradiance factor of a ring structure like B850 does not give a direct measure of the exciton size. An observed $L_{\mathrm{s}}$ can be the result of the delocalization length $L_{\mathrm{s}}$ or $N_{0}-L_{\mathrm{s}}$, where $N_{0}$ is the system size. This means that small $L_{\mathrm{s}}$ may imply either a highly localized or delocalized exciton. Additional measurements like the temperature dependence of $L_{\mathrm{S}}{ }^{18}$ or single molecule spectroscopy ${ }^{24}$ can resolve this ambiguity.

\section{Measures of the Exciton Delocalization Length}

The extent of delocalization of the optically generated exciton wave packet strongly influences the signals obtained in various optical measurements, and in order to fully understand the lightharvesting energy transfer mechanisms, it is important to characterize the properties of the basic excitations. Experimentally, $L_{\mathrm{d}}$ has been estimated from pump-probe spectra peaksplitting ${ }^{17}$ (the energetic difference between the excited-state absorption and the ground-state bleaching peaks), the absorbing dipole strength, ${ }^{25,32,54}$ and superradiance measurements. ${ }^{18,53-55}$ Theoretically, several tools have been proposed and used to estimate this property. ${ }^{15,16,23,29}$ Different authors have reported $L_{\mathrm{d}}$ values ranging from a few pigment molecules ${ }^{15-19}$ up to the full ring. ${ }^{31,32}$ Some of this discrepancy may be attributed to the use of different definitions. However, more importantly, different experimental techniques are expected to yield different coherence sizes. For instance, time-resolved and steady-state methods should give different results if the exciton delocalization length is time dependent. The exciton wave functions are linear combinations of molecular states, since electronic coupling causes molecules to behave coherently. The coherence can be reduced by static and dynamic disorder, which leads to localization. On the other hand, a short and spectrally broad excitation pulse creates a coherent superposition of a number of exciton states, i.e., an excitonic wave packet. Because of interference, the wave packet may have a major part of its amplitude in a very localized region of the aggregate. ${ }^{56}$ The coherence between exciton levels is rapidly destroyed due to dephasing and exciton relaxation. In some sense this can be seen as if an initially localized wave packet is delocalized by dephasing processes. However, we should keep in mind that the initially prepared excitonic wave packet (doorway state) may seem localized even if it has a precise phase-relationship between different molecular states over the entire aggregate. This illustrates the possible difficulties in discussing the interplay of coherence and localization.

A number of different definitions have been used for characterizing the exciton delocalization length. We reserve $L_{\mathrm{d}}$ for the generic exciton delocalization length and denote specific measures by different subscripts. Meier, Chernyak and Mukamel $^{15}$ considered the entire $\left|N_{\mathrm{mn}}(t)\right|$ (both diagonal and offdiagonal elements) as a distribution. The exciton delocalization 
length, was then defined as the inverse participation ratio associated with this distribution

$$
L_{\rho}(t)=\left[N_{0} \sum_{m n}\left|N_{m n}(t)\right|^{2}\right]^{-1}\left[\left(\sum_{m n}\left|N_{m n}(t)\right|\right)^{2}\right]
$$

In this expression, $N_{0}$ is the system size and the EDM is defined in eq 2.7. $L_{\rho}$ is a length scale on which the EDM decays along the anti-diagonal direction, and an intuitive measure that connects directly with experimental measurements.

Kühn and Sundström ${ }^{23}$ defined the exciton delocalization length $\left(L_{\mathrm{ks}}\right)$ as the full width half-maximum (fwhm) of the distribution function

$$
C_{n}(t) \equiv \sum_{m}\left|\left\langle N_{m, m+n}(t)\right\rangle_{\mathrm{disorder}}\right|
$$

This measures an autocorrelation distribution for site $n$ of the exciton density matrix, averaged over the coherence contributions from all other sites in the aggregate. $L_{\mathrm{ks}}$ is obtained by calculating the distribution from $n=0$ to 8 (for $\mathrm{B} 850$ of LH2) and taking the fwhm of this distribution. If the excitation is completely localized at one site (e.g., site 0 ), the distribution will be 1 at site 0 and exactly 0 for sites $1-8$, but if the excitation is more delocalized, the distribution will be broader according to the extent of coherence between the pigments. Furthermore, all one-exciton wave functions except for the lowest and highest (usually denoted $k=0$ and 9) are oscillatory and therefore the fwhm estimate will be increasingly difficult to obtain with increasing exciton delocalization. If the coherence between the exciton levels is dephased, this definition corresponds exactly to the autocorrelation definition used in refs 18 and 30 .

The participation ratio is based on general properties of distribution functions. The localization characteristics of a disordered system can be described using a set of functions based on the sum over the density distribution of the wave function ${ }^{57-60}$

$$
I_{\mathrm{n}}=\sum_{i}\left|\varphi_{i}\right|^{2 n}
$$

where $\varphi_{i}$ is the wave function for the $i$ th exciton level for a one-dimensional discrete lattice. This quantity may be averaged over the Boltzmann distribution of exciton states

$$
I_{\mathrm{n}}=\sum_{i}\left(\frac{P_{i}}{Z}\right)\left|\varphi_{i}\right|^{2 n}
$$

$I_{1}=1$ whereas $1 / I_{2}$, known as the inverse participation ratio, is a measure of the width of the distribution and can be used as an estimate of the exciton delocalization length.

Leegwater ${ }^{16}$ defined the exciton delocalization length as a generalized inverse participation ratio

$$
L_{\mathrm{gr}}{ }^{-1}=\sum_{i, \alpha, \alpha^{\prime}}\left|\left\langle i \mid \varphi_{\alpha}\right\rangle\right|^{2} \sqrt{\frac{P_{\alpha}}{Z}} \frac{\Gamma^{2}}{\Gamma^{2}+\left(\omega_{\alpha}-\omega_{\alpha^{\prime}}\right)^{2}}\left|\left\langle i \mid \varphi_{\alpha^{\prime}}\right\rangle\right|^{2} \sqrt{\frac{P_{\alpha^{\prime}}}{Z}}
$$

where $\varphi_{\alpha}$ is the exciton wave function and $\Gamma$ is the exciton dephasing rate. $P_{\alpha}=\exp \left(-\epsilon_{\alpha} / k_{B} T\right)$ is the Boltzmann factor of state $\alpha$ and $Z=\sum_{\alpha} \exp \left(-\epsilon_{\alpha} / k_{\mathrm{B}} T\right)$ is the canonical partition function where $k_{\mathrm{B}}$ is the Boltzmann constant.

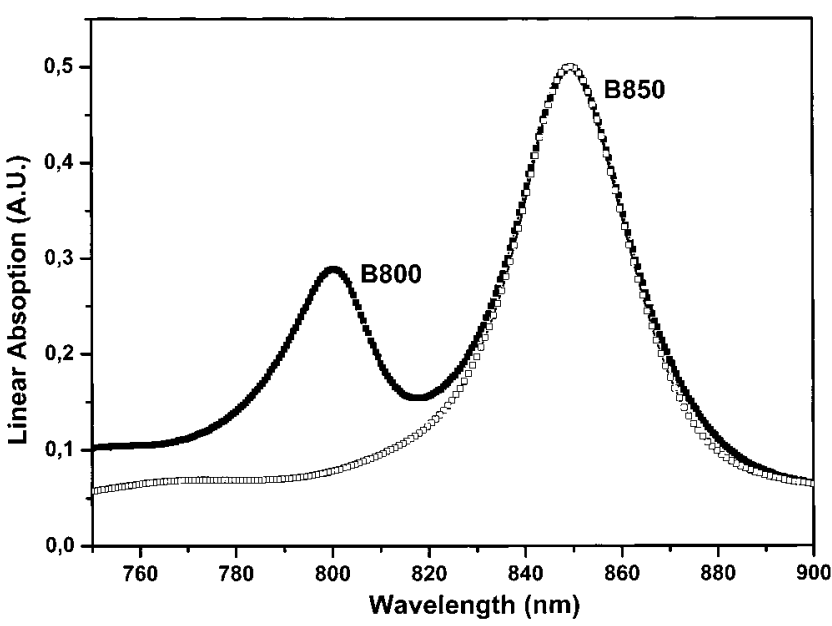

Figure 2. Linear absorption spectra of LH2 from Rps. acidophila at room temperature: (open circles) experimentally recorded; (filled squares) simulated absorption spectra for an inhomogeneous broadening of $250 \mathrm{~cm}^{-1}$, a homogeneous line width of $75 \mathrm{~cm}^{-1}$, and the first excited molecular state at $12426 \mathrm{~cm}^{-1}$.

The ordinary inverse participation ratio, ${ }^{29}$ a special case of $L_{\mathrm{gr}}$, has been extensively used for light-harvesting systems..$^{20,26,30}$ By setting $\Gamma=0$, eq 3.5 yields

$$
L_{\mathrm{ipr}}{ }^{-1}=\sum_{i, \alpha}\left|\left\langle i \mid \varphi_{\alpha}\right\rangle\right|^{4} \frac{P_{\alpha}}{Z}
$$

It is straightforward to add time dependence to the definitions of $L_{\mathrm{gr}}$ and $L_{\mathrm{ipr}}$ by including the population dynamics of the exciton states $\left(P_{\alpha}(t)\right)$. However, inter-exciton-level coherence and dephasing are not accounted for in these two definitions.

\section{Application to the B850 System}

We describe the B850 antenna by a model aggregate made out of 18 three-level systems with dipole-dipole coupling. Existing literature does not provide unique values for the characteristics of these systems. A wide range of interaction strengths has been proposed for the B850 aggregate, ranging from 200 up to $800 \mathrm{~cm}^{-1}$. Accumulating evidence suggests a nearest neighbor interaction of about $300 \mathrm{~cm}^{-1}$; for a review see ref 30 . We have estimated the interaction energies using the structure of Rps. acidophila and assuming dipole-dipole interaction. The B850 aggregate consists of a slightly dimerized ring-structure where the nearest neighbor interactions alternate between $J=340$ and $230 \mathrm{~cm}^{-1}$. All other pairwise interaction terms are included, but they are all less or equal to $46 \mathrm{~cm}^{-1}$. The disorder was drawn from a Gaussian distribution function

$$
P\left(\epsilon_{n}\right)=\frac{1}{\sigma \sqrt{2 \pi}} \exp \left(-\epsilon_{n}^{2} / 2 \sigma^{2}\right)
$$

where the distribution of $\epsilon_{n}$ represent the uncorrelated static fluctuations of the energy levels caused by the surrounding protein cage, and $\sigma$ is the variance of this distribution. We used a width $\left(\sigma_{\text {inh }}\right)$ of the inhomogeneous broadening (energetic disorder) of $255 \mathrm{~cm}^{-1}$ (variance); see Figure 2. This disorder is in agreement with values used in other recent works. ${ }^{15,18,30}$ The homogeneous (the inverse of dephasing constant), $\Gamma=75 \mathrm{~cm}^{-1}$, and the transition energy for the first excited molecular state $\left(\mathrm{S}_{0} \rightarrow \mathrm{S}_{1}\right), E_{i}=12426 \mathrm{~cm}^{-1}$, were estimated by comparing the calculated linear and transient absorption spectra with the experimental room-temperature spectra. The assignment of the 

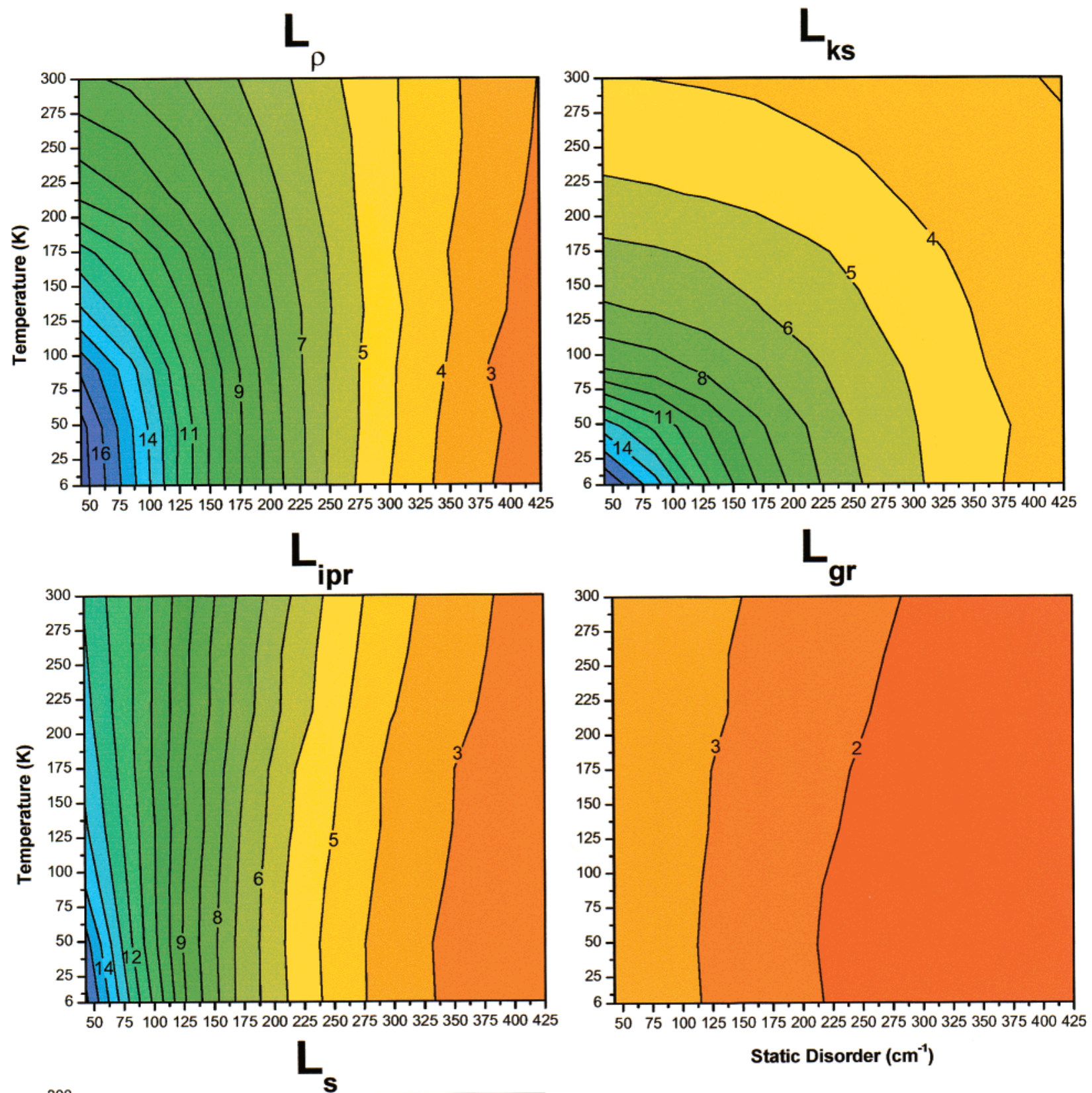

Static Disorder $\left(\mathrm{cm}^{-1}\right)$
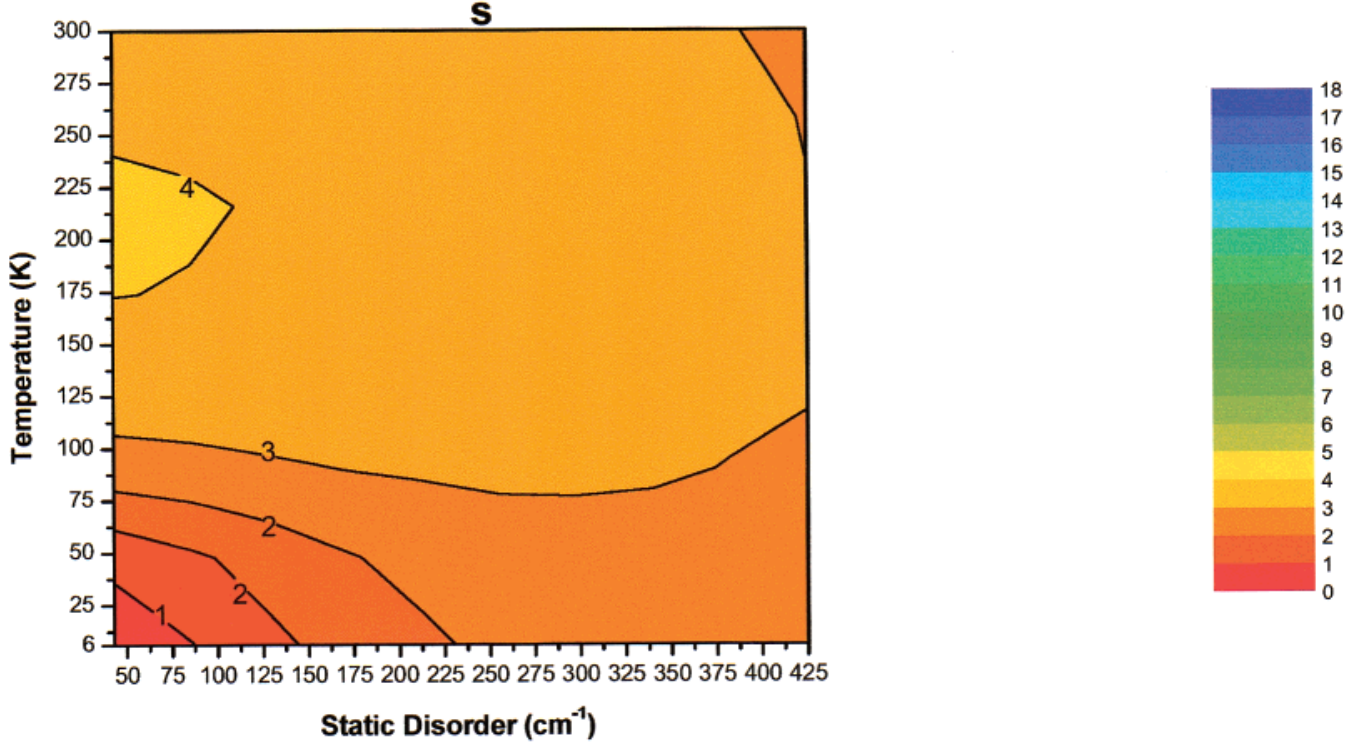

Figure 3. Panels displaying the result from the corresponding definitions of $L_{\mathrm{d}}$ given in section III. In all cases, the exciton was calculated in the thermalized state. 
homogeneous and inhomogeneous broadening ${ }^{14}$ is based on the separation of the bath fluctuation time scales. We find that the parameters used here are within the ranges used in the literature. We point out that at higher temperatures the borderline between homogeneous and inhomogeneous parts of the spectrum is not very clear cut and one needs to consider the relevant time scales of the experimental observables which are used in the analyzes. Rigorous treatment of the temperature dependence of spectra goes beyond the scope of the current article, where we have included the temperature via the Redfield relaxation matrix.

The characteristics of the second excited transition $\left(S_{1} \rightarrow S_{2}\right)$ are less known. On the basis of ref 61, we have used an energetic offset $(\Delta)$ between the $\left(S_{1} \rightarrow S_{2}\right)$ and the $\left(S_{0} \rightarrow S_{1}\right)$ transitions of $\sim 500 \mathrm{~cm}^{-1}$ and further assumed that the respective transition dipoles are parallel and the ratio $\kappa=\mu^{(1 \rightarrow 2)} / \mu^{(0 \rightarrow 1)}$ is 0.6 . All parameters were further verified by comparing calculated and measured transient absorption spectra (see below).

We consider the pulse duration to be short compared to the nuclear dynamics, but long compared to the electronic dephasing time scale. The snapshot doorway wave packet ${ }^{14}$ was convoluted with the Gaussian spectral profile of the excitation pulse,

$$
\mid\left(\omega_{1}-\bar{\omega}_{1}\right)=\frac{1}{\sigma_{1} \sqrt{2 \pi}} \exp \left(-\left(\omega_{1}-\bar{\omega}_{1}\right)^{2} / 2 \sigma_{1}^{2}\right)
$$

where $\bar{\omega}_{1}$ is the pulse central frequency with a variance of 151 $\mathrm{cm}^{-1}$ for the pump pulse (which corresponds to a Gaussian pulse with a fwhm duration of $\sim 40 \mathrm{fs}$ ).

In ref 26 the reorganization energy $(\lambda)$ of the B850 aggregate was estimated using the three pulse photon echo peak shift (3PEPS) experiment to be about $40 \mathrm{~cm}^{-1}$. In order for the Redfield theory to yield relaxation and dephasing rates comparable with experiment, we used a reorganization energy of $130 \mathrm{~cm}^{-1}$. This difference is most likely due to our bath model, consisting of a single overdamped Brownian oscillator per chromophore. In addition, the aggregate reorganization energy reported in ref 26 is different from the molecular reorganization energy used here. We assumed an inverse nuclear relaxation time $(\Lambda)$ of $1300 \mathrm{~cm}^{-1}$. These parameters satisfy the necessary condition, $\Lambda / \lambda \gg 1$ required for the applicability of the Redfield equations.

The parameters above will now be used to analyze the exciton coherence size in LH2. The various measures of $L_{\mathrm{d}}$ are very similar in the two limiting cases, i.e., $L_{\mathrm{d}} \sim 18$ for zero temperature without disorder and $\sim 1$ in the other extreme limit. However, for the generalized inverse participation ratio $\left(L_{\mathrm{gr}}\right)$ and the superradiance factor $\left(L_{\mathrm{s}}\right)$ the analysis is more subtle. For the former definition, one needs to let $\Gamma \rightarrow 0$ in order for $L_{\mathrm{gr}}$ to approach 18 (doing so reduces the definition to that of the ordinary inverse participation ratio). The superradiance factor $\left(L_{\mathrm{s}}\right)$ for a ringlike structure goes to zero at low temperature without disorder (see below).

Figure 3 displays the dependence of $L_{\mathrm{d}}$ on temperature and disorder for the various different definitions. At room temperature, the thermalized Kühn-Sundström length, $L_{\mathrm{ks}}$, is about 4 $\mathrm{BChl}$ pigments, in good agreement with previously reported results for a static disorder with a Gaussian profile and a variance of about $210 \mathrm{~cm}^{-1} .^{23}$ As can be seen from the figure, this definition of $L_{\mathrm{d}}$ depends quite strongly on both temperature and inhomogeneous broadening. $L_{\rho}$ results in a qualitatively similar behavior to $L_{\mathrm{ks}}$, but it decreases much more slowly with increasing temperature and disorder. A well-defined value of $L_{\rho}$ can be calculated under all conditions, even when the coherence between exciton levels is large. At room temperature in the thermalized limit $L_{\rho}$ is about $6 \mathrm{BChl}$ pigments, slightly larger than $L_{\mathrm{ks}}$. $L_{\mathrm{ipr}}$, and $L_{\mathrm{gr}}$, both show a very weak temperature dependence but a strong dependence on disorder. A reasonable static disorder of $250 \mathrm{~cm}^{-1}$ at room temperature results in $L_{\mathrm{ipr}}$ $\sim 4-5 \mathrm{BChl}$ molecules, whereas the generalized inverse participation ratio $\left(L_{\mathrm{gr}}\right)$ gives around 2 pigments. In addition, $L_{\mathrm{gr}}$ has a strong dependence on the exciton dephasing rate $\Gamma$, which causes a decrease of the delocalization length at all temperatures and disorder realizations.

The superradiance factor $\left(L_{\mathrm{S}}\right)$ is displayed in Figure 3 as well. $L_{\mathrm{S}}$ decreases as the temperature and disorder is decreased, in contrast to the behavior of the delocalization lengths discussed above. This behavior reflects the specific geometry of the emitting dipoles included in the definition of $L_{\mathrm{s}}$ (eq 2.8). Since the lowest exciton state is optically forbidden (weakly allowed when disorder is included) for the ringlike symmetry of B850, $L_{\mathrm{s}}$ will decrease at low temperatures when this state becomes highly populated. $L_{\mathrm{s}}$ is estimated to be $\sim 3$ at room temperature for $250 \mathrm{~cm}^{-1}$ and as the temperature is decreased $L_{\mathrm{s}}$ decreases slowly to roughly 2 at $6 \mathrm{~K}$. Superradiance of B850 was measured by Monshouwer et al. in the temperature range from 6 to 300 $\mathrm{K} ;{ }^{18}$ the superradiance factor was found to be $\sim 3-4 \mathrm{BChls}$ in this temperature range. With the same parameters used to fit the transient absorption spectrum $\left(\sigma_{\text {inh }}=250 \mathrm{~cm}^{-1}, \Gamma=75\right.$ $\mathrm{cm}^{-1}$ ) our calculations result in a very similar behavior of $L_{\mathrm{s}}$, i.e., $L_{\mathrm{S}} \sim 2.5$ at low temperature $(<77 \mathrm{~K})$ and $L_{\mathrm{s}} \sim 3.2$ at 300 $K$. The superradiance measurements of course yield the thermalized value of the $L_{\mathrm{s}}$, since this is a time-integrated technique.

To study the temporal evolution of the delocalization length, we fixed the disorder at $210 \mathrm{~cm}^{-1}$ and calculated $L_{\mathrm{d}}$ for a series of time delays at $300 \mathrm{~K}$ (see Figure 4, panel A), using the three definitions that provide a time dependence. $L_{\mathrm{d}}$ is quite large at short times $(\sim 5-13)$ and decreases rapidly to $\sim 4-6$ sites. Note, however, that $L_{\mathrm{ks}}$ at early times is most likely underestimated due to the difficulty in determining the fwhm of the distribution when the coherence between exciton levels is large (see previous section). The calculations show that the exciton dephasing is completed in a few hundred femtoseconds. $L_{\rho}$ has a slower decay than the other two definitions of $L_{\mathrm{d}}$, due to the slow population redistribution predicted in this case, also seen in the timedependent DM plot (panel B).

The localization process can be qualitatively described in a few steps. At time zero, $L_{\mathrm{d}}$ is determined by static properties such as the inhomogeneous broadening and the extent of the coherence between exciton levels created by the pulse. The decrease that takes place at later times is caused by the excitonphonon interaction. The rapid initial decay seen in panel A can be understood by studying panels B, C, and D, which show the time-dependent EDM elements using the same parameters as in panel A. The rapid decrease in panel A can be assigned mainly to the destruction of the initially prepared coherence between the exciton levels and the initial fast inter exciton level relaxation. The dynamics above $\sim 250$ fs reflect the slower part of the population relaxation. The final thermalized size of $L_{\mathrm{d}}$ depends on the combination of the different static parameters such as temperature, disorder, and exciton dephasing.

Time-resolved transient absorption spectra, measured at both room temperature and $77 \mathrm{~K}$ with $\sim 100 \mathrm{fs}$ pulses, ${ }^{42}$ suggest a spectral evolution on a few hundred femtosecond time scale that may be attributed to the processes leading to exciton localization. We have fitted both the linear absorption spectrum (Figure 2) and the transient spectra (Figure 5), using the same parameter values. Comparing the measured and the calculated 

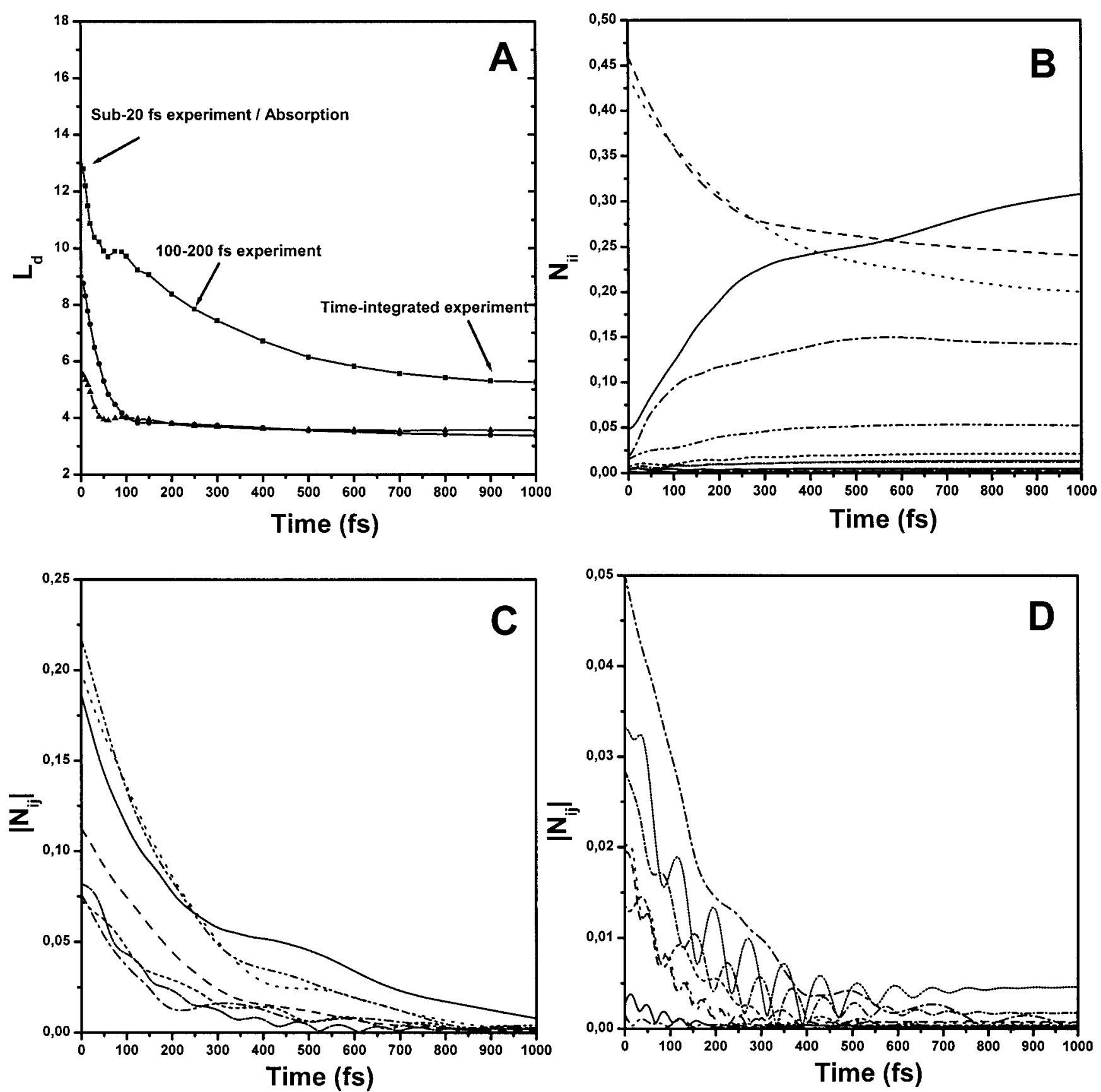

Figure 4. (A) Time evolution of $L_{\rho}$ (squares), $L_{\mathrm{s}}$ (circles), and $L_{\mathrm{ks}}$ (triangles). The inhomogeneous disorder has a variance of $250 \mathrm{~cm}^{-1}$ and the spectral density parameters $\lambda=130 \mathrm{~cm}^{-1}$ and $\Lambda=1300 \mathrm{~cm}^{-1}$. (B) The 18 population $\left(N_{i i}(t)\right)$ elements. (C, D)Amplitude of the largest and the smaller, respectively, off-diagonal coherence elements $\left(\left|N_{i j}(t)\right|\right)$. The time-dependent exciton density matrix elements were calculated at room temperature and with one realization of Gaussian disorder. The pump pulse was tuned to the middle of the one-exciton band, $850 \mathrm{~nm}$.

transient absorption spectra at $200 \mathrm{fs}, 500 \mathrm{fs}$, and 2 ps (Figure 5) strongly suggests that the spectral evolution in fact reflects exciton relaxation and that the B850 exciton evolves from an initially large $L_{\mathrm{d}}$ to a substantially smaller size. This evolution is basically completed in $\sim 1 \mathrm{ps}$, which is also evident from the time dependence of $L_{\rho}$ (Figure 4). The faster decay of $L_{\mathrm{ks}}$ and $L_{\mathrm{S}}$ is due to the above-mentioned interference problem in the first case and the weak population dependence of the latter. Recent experiments on B850 with high time resolution ( 20 fs $)^{25}$ also suggest a large exciton size at early time and exciton localization on the time scale of a few hundred femtoseconds.

Finally, in Figure 6 we display the variation of the initial delocalization length with the pump-pulse wavelength between 870 and $810 \mathrm{~nm}$ for three different pulse lengths. We point out that we are using the time-domain snapshot limit, which means that we do not calculate the system evolution during the pulses. Various pulse lengths were simulated via different spectral widths of the excitation pulse. A shorter pulse generally creates a larger $L_{\mathrm{d}}$ because of the additional delocalization due to the coherence between exciton levels. The differences between $L_{\mathrm{d}}$ 's obtained with 200 fs and 1 ps pulses are negligible since the relatively narrow spectral width of the pulses almost selectively excite only one exciton state and very little inter-exciton coherence is created. $L_{\mathrm{ks}}$ does not show any significant dependence on pulse properties, which is partly related to the difficulties with exciton state interference.

Our results imply that the variation of $L_{\mathrm{d}}$ values of B850 reported in the literature results from the use of different 

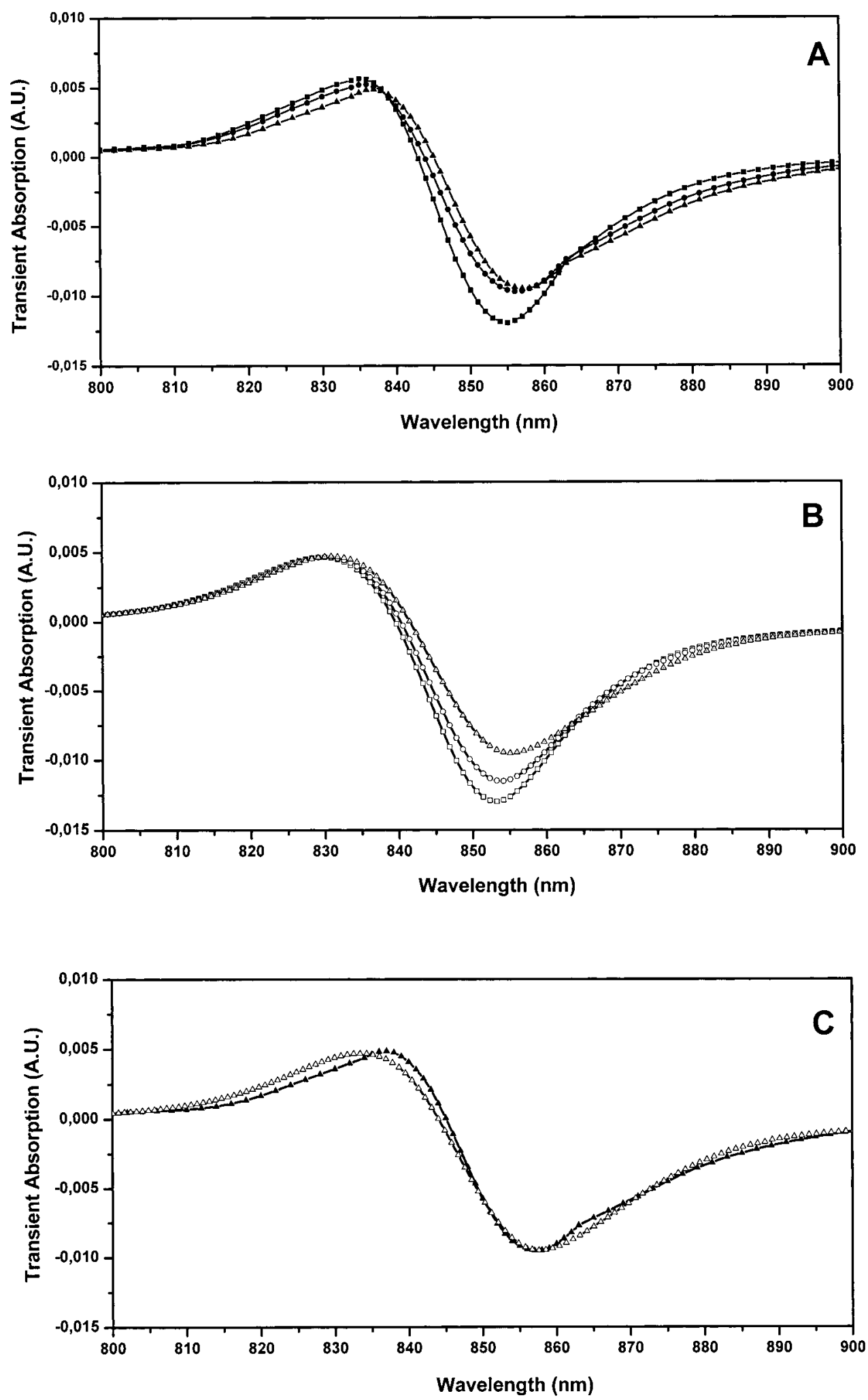

Figure 5. (A) Experimental pump-probe spectra for three different time delays (squares, $200 \mathrm{fs}$; circles, $500 \mathrm{fs}$; triangles, $2.0 \mathrm{ps}$ ). (B)Simulated spectra for the same time delays as in panel A and with the following parameters: second excited molecular state parameters $\kappa=0.6$ and $\Delta=500$ $\mathrm{cm}^{-1}$, respectively; $\Gamma=75 \mathrm{~cm}^{-1}, \sigma_{\mathrm{inh}}=250 \mathrm{~cm}^{-1}$. (C) Comparison of the simulated (open triangles) with the experimental (filled triangles) spectra at 2 ps time delay.

techniques and time resolution. Thus, a technique with a time resolution of $<25$ fs or a technique probing the instantaneous spectral properties should be able to capture the initial size of the exciton wave packet (see also the $L_{\mathrm{d}}$ dynamics illustrated in Figure 4A). On the other hand, a $\sim 100$ fs time-resolved measurement of the kind reported by many authors ${ }^{30,42}$ captures some of the evolution but does not provide the initial value of $L_{\mathrm{d}}$. A steady-state superradiance measurement integrates $L_{\mathrm{d}}$ over the fluorescence lifetime $(\sim 1 \mathrm{~ns})$ of the aggregate and thus provides the thermalized value. Of course, in principle, spontaneous emission could be measured with a time resolution adequate to resolve the localization process. No such measure- 


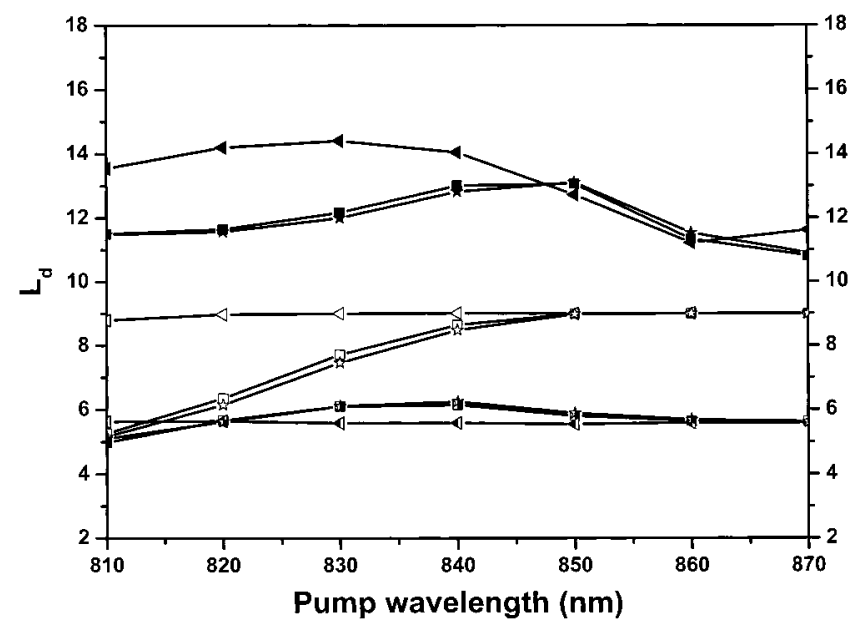

Figure 6. Initial $L_{\mathrm{d}}$ presented for three different pulse durations as a function of wavelength: (filled symbols) $L_{\rho}$; (open symbols) $L_{s}$; (semifilled symbols) $L_{\mathrm{ks}}$. The different types of symbols correspond to a fwhm of the pulse durations: (triangles) $20 \mathrm{fs}$; (squares) $200 \mathrm{fs}$; (stars) $1 \mathrm{ps}$.

ment has so far been reported. These three different types of experiments are indicated by the arrows in Figure 4, panel A.

\section{Conclusions}

We have compared the calculated transient absorption spectra to the measured spectra for different time delays. We showed that the spectral dynamics are qualitatively reproduced, both relaxation time scales and spectral evolution. The spectral dynamics correspond to localization of the exciton from an initial substantially delocalized exciton wave packet to a significantly smaller thermalized exciton and occurs on a time scale of a few hundred femtoseconds. We have compared several definitions that provide different estimates of $L_{\mathrm{d}}$ for given disorder parameters and temperature. $L_{\rho}$ and $L_{\mathrm{ks}}$ are constructed using the EDM and sampling over the coherence contributions, but weight these contributions differently, resulting in different dependencies on temperature and disorder. $L_{\rho}$ is for most parameter values significantly larger than $L_{\mathrm{ks}} . L_{\mathrm{ipr}}$ and $L_{\mathrm{gr}}$ display similar temperature and disorder dependencies, the former is weak while the latter is much more pronounced. Finally, we note that in this paper the exciton-phonon coupling is considered in the weak-coupling limit which introduces transitions between exciton levels (exciton relaxation leading to thermalization) but does not modify the exciton wave functions. The latter would occur in the case of strong electron-phonon interaction, which leads to polaron formation. ${ }^{42,46,52}$

Acknowledgment. We thank Dr. J. L. Herek for critically reading and commenting on the manuscript, Dr. T. Polivka for the experimental data, and Dr. V. Chernyak for enlightening discussions. This work was financially supported by the Crafoord foundation and NFR. S.M. gratefully acknowledges the support of The National Science Foundation and the Petroleum Research Fund administered by the American Chemical Society.

\section{References and Notes} 996.

(1) J-aggregates; Kobayashi, T., Ed.; World Scientific: Singapore,

(2) Tretiak, S.; Chernyak, V.; Mukamel, S. Phys. Rev. Lett 1996, 77, 4656.

(3) Sundström, V.; Pullerits, T.; van Grondelle, R. J. Chem. Phys. B 1999, 103, 2327.
(4) Tretiak, S.; Chernyak, V.; Mukamel, S. J. Chem. Phys. B 1998, 102,3310 .

(5) Nakano, M.; Takahata, M.; Fujita, H.; Kiribayashi, S.; Yamaguchi, K. Chem. Phys. Lett. 2000, 323, 249.

(6) Frenkel, J. I. Phys. Rev. 1931, 37, 17.

(7) Davydov, A. S. Theory of Molecular Excitons; Plenum Press: New York, 1971

(8) Ziman, J. M. Models of Disorder; Cambridge University Press: Cambridge, 1979.

(9) de Bree, P.; Wiersma, D. A. J. Chem. Phys. 1979, 70, 790

(10) Pearlstein, R. M.; Hemenger, R. P. Proc. Natl. Sci. U.S.A. 1978, 75,4920 .

(11) McDermott, G.; Prince, S. M.; Freer, A. A.; HawthornthwaiteLawless, A. M.; Papiz, M. Z.; Cogdell, R. G.; Isaacs, N. W. Nature 1995, $374,517$.

(12) Koepke, J.; Hu, X.; Muenke, C.; Schulten, K.; Michel, H. Structure 1996, 4, 581 .

(13) Pullerits, T.; Hess, S.; Herek, J. L.; Sundström, V. J. Phys. Chem. B 1997, 101, 10560 .

(14) Mukamel, S. Principles of Nonlinear Optical Spectroscopy; Oxford University Press: New York, 1995.

(15) Meier, T.; Chernyak, V.; Mukamel, S. J. Phys. Chem. B 1997, 101, 7332. Zhang, WM.; Meier, T.; Chernyak, V.; Mukamel, S. J. Chem. Phys. 1998, 108, 7763.

(16) Leegwater, J. A. J. Chem. Phys. 1996, 100, 14403.

(17) Pullerits, T.; Chachisvilis, M.; Sundström, V. J. Chem. Phys. 1996, $100,10787$.

(18) Monshouwer, R.; Abrahamsson, M.; van Mourik, F.; van Grondelle, R. J. Phys. Chem. B 1997, 101, 7241.

(19) Ray, J.; Makri, N. J. Phys. Chem. A 1999, 103, 9417.

(20) Novoderezkhin, V.; Monshouwer, R.; van Grondelle, R. J. Chem. Phys. B 1999, 103, 10540.

(21) Meier, T.; Zhao, Y.; Chernyak, V.; Mukamel, S. J. Chem. Phys. 1997, 107, 3876.

(22) Bakalis, L. D.; Knoester, J. J. Phys. Chem. B 1999, 103, 6620.

(23) Kühn, O.; Sundström, V. J. Chem. Phys. 1997, 107, 4154.

(24) Zhao, Y.; Meier, T.; Zhang, W. M.; Chernyak, V.; Mukamel, S. J. Phys. Chem. B 1999, 103, 3954

(25) Book, L. D.; Ostafin, A. E.; Ponomarenko, J. R.; Scherer, N. F. J. Phys. Chem. B 2000, 104, 8295. Book, L. D.; Ostafin, A. E.; Ponomarenko, N.; Norris, J. R.; Scherer, N. F.; Mukamel, S. In Ultrafast Phenomena XII; Elsasser, T., Mukamel, S., Murnane M., Scherer, N., Eds.; SpringerVerlag: Berlin, 2000; p 527.

(26) Jimenez, R.; van Mourik, F.; Yu, J. Y.; Fleming, G. R. J. Phys. Chem. B 1997, 101, 7350 .

(27) Kennis, J. T. M.; Streltsov, A. M.; Permentier, H.; Aartsma, T. J.; Amesz, J. J. Phys. Chem. B 1997, 101, 8369.

(28) Nagarajan, V.; Johnson, E. T.; Williams, J. C.; Parson, W. W. J. Phys. Chem. B 1999, 103, 2297.

(29) Fidder, H. Thesis, Groningen University 1993.

(30) Chachisvilis, M.; Kühn, O.; Pullerits, T.; Sundström, V. J. Phys. Chem. B 1997, 101, 7275 .

(31) Nagarajan, V.; Alden, R. G.; Williams, J. C.; Parson, W. W. Proc. Natl. Acad. Sci. U.S.A. 1996, 93, 13774.

(32) Leupold, D.; Stiel, H.; Teuchner, K.; Nowak, F.; Sandner, W.; Ücker, B.; Scheer, H. Phys. Rev. Lett. 1996, 77, 4675.

(33) Wu, H. M.; Small, G. J. Chem. Phys. 1997, 218, 225. Wu, H. M.; Ratsep, M.; Lee, I. J.; Cogdell, R. J.; Small, G. J. Phys. Chem. B 1997 101, 7654. Wu, H. M.; Small, G. J. Phys. Chem. B 1998, 102, 888.

(34) Mukamel, S. In Nonlinear Molecular Optics; Zyss, J., Ed.; Academic Press: New York, 1994; pp 1-46.

(35) Dubovsky, O.; Mukamel, S. J. Chem. Phys. 1991, 95, 7828.

(36) Leegwater, J. A.; Mukamel, S. Phys. Rev. A 1992, 46, 452.

(37) Knoester, J.; Mukamel, S. Phys. Rep. 1991, 205, 1.

(38) van Oijen, A. M.; Ketelaars, M.; Köhler, J.; Aartsma, T. J.; Schmidt, J. Science 1999, 285, 400.

(39) Mostovoy, M. V.; Knoester, J. J. Phys. Chem. B 2000, 104, 12355.

(40) Mukamel, S.; Tretiak, S.; Wagersreiter, T.; Chernyak, V. Science 1997, 277, 781.

(41) Spano, F. C.; Kuklinski, J. R.; Mukamel, S. Phys. Rev. Lett. 1990, 65, 211. Spano, F. C.; Kuklinski, J. R.; Mukamel, S. J. Chem. Phys. 1991, 94, 7534.

(42) Polivka, T.; Pullerits, T.; Herek, J. L.; Sundström, V. J. Phys. Chem. B 2000, 104, 1088.

(43) Blum, K. Density Matrix Theory and Applications; Plenum Press: New York, 1981.

(44) Dahlbom, M.; Minami, T.; Chernyak, V.; Pullerits, T.; Sundström, V.; Mukamel, S. J. Phys. Chem. B 2000, 104, 3976.

(45) Chernyak, V.; Zhang, W. M.; Mukamel, S. J. Chem. Phys. 1998, $108,9587$.

(46) Chernyak, V.; Mukamel, S. J. Chem. Phys. 1996, 105, 4565.

(47) Redfield, A. G. IBM J. Res. 1957, 1, 19. 
(48) Pollard, W. T.; Felts, A. K.; Friesner, R. A. Adv. Chem. Phys. 1997, 93, 77. Pollard, W. T.; Friesner, R. A. J. Chem. Phys. 1994, 100, 5054. (49) Jean, J. M.; Friesner, R. A.; Fleming, G. R. J. Chem. Phys. 1992, 96, 5827.

(50) Mukamel, S.; Ciordas-Ciurdariu, C.; Khidekel, V. IEEE 1996, 32, 1278.

(51) Mukamel, S. J. Chem. Phys. 1997, 107, 4165.

(52) Feynman, R. P. Statistical Mechanics; Perseus Books: Reading, MA, 1998

(53) Chernyak, V.; Meier, T.; Tsiper, E.; Mukamel, S. J. Phys. Chem. B 1999, 103, 10294 .

(54) Kennis, J. T. M.; Aartsma, T. J.; Amesz, J. Biochim. Biophys. Acta 1994, 188, 278.
(55) Owen, G. M.; Hoff, A. J.; Jones, M. R. J. Chem. Phys. B 1997, $101,7197$.

(56) Knox, R. S.; Gülen, D. Photochem. Photobiol. 1993, 57, 40.

(57) Fyodorov, Y. V.; Mirlin, A. D. Phys. Rev. B 1995, 51, 13403.

(58) Hall, M. J. W. Phys. Rev. A 1999, 59, 2602.

(59) Plerou, V.; Gopikrishan, P.; Rosenow, B.; Nunes Amaral, L. A.; Stanley, H. E. Phys. Rev. Lett. 1999, 83, 1471.

(60) The function $I_{2}$ is in parts of the literature referred to as the inverse participation ratio, but here we use that designation for $I_{2}{ }^{-1}$.

(61) Martinsson, P.; Sundström, V.; Åkesson, E. FEBS 2000, 465, 107. Martinsson, P.; Thesis, Lund University, 1999. 